\title{
Pain and chronic pain epidemiology
}

\author{
Implications for clinical and public health fields
}

\author{
Thomas E. Dorner
}

Received: 6 December 2017 / Accepted: 8 December 2017 / Published online: 21 December 2017

(c) Springer-Verlag GmbH Austria, part of Springer Nature 2017

Undoubtedly, pain is one of the most common symptoms in health care settings, leading to loss of function and decline in quality of life that puts enormous pressure on the affected individuals; however, pain, especially chronic, presents not only a problem for individuals, but also for the whole community, and is therefore a major public health concern.

\section{Epidemiology of chronic pain}

Epidemiology, as defined by the World Health Organization is: "the study of the distribution and determinants of health-related states or events (including disease), and the applications of this study to the control of diseases and other health problems" [1]. Thus, epidemiology is not only about collecting numbers, but also takes into account the consequences of quantified indicators, which means prevention. In the case of chronic pain, prevention not only means prevention of the disease that brought about chronic pain, but also the consequences, i. e. loss of function and productivity, work disability, mental disorders, sexual discontent, inappropriate health care utilization, etc. [2]. Therefore, epidemiology is necessary to quantify a health hazard in a certain population, to prioritize efforts in health care, and to improve preventive and clinical management, limit disease severity and minimize disability. This edition of the "Wiener klinische Wochenschrift" presents one of the very few population-based studies showing the epidemiology of pain and chronic pain in the general Austrian population,

\footnotetext{
T. E. Dorner (凶)

Center for Public Health, Department of Social and Preventive Medicine, Unit Lifestyle and Prevention, Medical University of Vienna, Kinderspitalgasse 15/1, 1090 Wien, Austria

thomas.dorner@meduniwien.ac.at
}

based on the Austrian Health Interview Survey, which is composed of a remarkable sample size of more than 15,000 subjects [3]. This study shows a 1-year prevalence of severe pain of $38.6 \%$ and of chronic pain of $24.9 \%$ in the general Austrian population older than 15 years [3].

\section{Epidemiological data and clinical needs}

Scientific presentations and stakeholder discussions about pain often begin with epidemiological figures and follow the paradigm: the higher the numbers, the higher the burden for the affected people, the higher the costs for society, and the higher priorities should be given to the problem in the health care planning and financing. These facts often misguide researchers to present the highest numbers that can be derived from an epidemiological study. The question remains: what clinical impact do these epidemiological numbers have on chronic pain? Does every patient affected with chronic pain need to be treated in specialized or highly specialized health care settings, such as interdisciplinary pain centers or university clinics? The data presented in the study published in this issue [3] are population-based, and population-based data on chronic pain usually show a higher prevalence than routinely collected clinical data. This is due to the fact that pain is a feature of many health-related conditions that are coded and classified as separate diseases, although pain, especially chronic pain is acknowledged as a condition in its own right. Additionally, many patients with chronic pain do not seek professional help (but do self-care) or are only treated in primary care (with very limited or no documentation of pain in official health statistics). Therefore, numbers of population-based surveys present more realistic data on the frequency of pain than numbers from the health care system; however, it remains unclear if 
medical treatment of chronic pain in the health sector for patients without relevant burden of suffering and functional limitations is necessary. It has been argued that a distinction should be made between persons who are affected by pain and who can easily cope on their own, and patients with "pain disease", i. e. with high pain intensity and additional impairment due to elevated degrees of anxiety or depression related to pain, for which treatment in the health care setting is necessary [4]. A prerequisite that persons suffering from chronic pain can deal with their medical condition on their own, is empowerment, giving them skills in patient self-management, and involve them in pain management decision-making together with the health care provider [5]. In fact, another Austrian study in this edition has shown that a higher degree of health literacy in patients with chronic pain is associated with lower pain intensity and lower disability due to pain [6]; therefore, more emphasis on improving health literacy and more courage towards empowering patients with chronic pain towards selfmanagement is necessary.

\section{Pain in the biopsychosocial context}

A publication on pain and chronic pain epidemiology in this edition also reveals that low education, unemployment status, lack of social support, and especially depression and anxiety are associated with pain prevalence and with chronicity [3]. This is in line with previous epidemiological studies on chronic pain where modifiable and unmodifiable risk factors for chronic pain were found [7]. Knowing these risk factors, even if unmodifiable, is important to identify, design and target relevant person-tailored interventions in chronic pain as well as to prevent various adverse outcomes in patients with chronic pain [7]. The findings are also in accordance with the more and more acknowledged biopsychosocial model of (chronic) pain [5]. Biological factors associated with chronic pain include tissue damage and diseases, pain intensity and quality, physical functional interference and sleep disturbance. Psychological factors associated with chronic pain include emotional distress, attention deficit, attitudes and beliefs, coping skills, pain catastrophizing, and kinesiophobia. Social factors associated with pain include interpersonal relationships, working ability and working disability, cultural aspects and environmental factors [5]. These biological, psychological and social dimensions can greatly interfere with each other. For example, sleep disorders are very common in patients with chronic pain and two thirds report no recovering sleep due to chronic pain according to a further article in this edition [8]. Furthermore, sleep disorders have a biological dimension, are often associated with frequent mental disorders and affect social life, such as the ability to work and social relationships. Tackling the problem of sleep disturbance in patients with chronic pain is important, because sleep problems are known to worsen depression in patients with chronic pain, thus exacerbating a known risk factor for chronic pain; therefore, addressing sleep problems can reduce chronic pain, lessen the risk of depressive illness development and improve the quality of life [7].

\section{Implication of pain on society}

Work disability is one of the major problems for society that is associated with chronic pain and is also a part of the social dimension of pain. Chronic pain is one of the main reasons for work absenteeism, i. e. sickness absences and disability pensions. In this edition, one article shows that $32.9 \%$ of the employed Austrian population aged 15-65 years who are affected by severe pain, were on sick leave due to pain. Education, income, and type of employment were significantly associated with absenteeism due to pain, at given pain intensity, comorbidities and sociodemographic factors [9]; however, many people stay at the workplace despite chronic pain, with their working ability being affected. Furthermore, estimates show that this presenteeism due to chronic pain, leads to four times more hours of work disability than absenteeism due to chronic pain [10]. So, the problems for society concerning chronic pain are much higher than can be assumed from figures for work disability.

\section{Summary}

Tackling chronic pain is a problem which not only affects the clinical setting. As it reduces working ability and productivity, it affects society in its entirety. Increasing health literacy in the total population is one tool that can prevent chronic pain, lower pain intensity and limitations due to pain, and prevent unnecessary health care utilization due to pain. In the health care setting, tackling the problem of chronic pain goes beyond pain treatment itself. Chronic pain is complex and comprises biological, psychological and social aspects, can be associated with frequent mental disorders, sleep disturbances as well as sociodemographic and socioeconomic factors. Many of those factors need to be managed more than to be cured; therefore, chronic pain often demands a patient-centered, integrated care with multiprofessional teams, in which the patient and not the disease is the focus of treatment, also allowing the patients themselves to be a part of this team and empowered towards selfmanagement.

Conflict of interest T.E. Dorner declares that he has no competing interests. 


\section{References}

1. World Health Organization. 2017. http://www.who.int/ topics/epidemiology/en/. Accessed Dec. 20, 2017.

2. Dorner TE, Crevenna R. Preventive aspects regarding back pain. Wien Med Wochenschr. 2016;166(1-2):15-21.

3. Dorner TE, Stein KV, Hahne J, Wepner F, Friedrich M, Mittendorfer-Rutz E. How are socio-demographic and psychosocial factors associated with the prevalence and chronicity of severe pain in 14 different body sites? A cross-sectional population-based survey. Wien Klin Wochenschr. 2017;129. https://doi.org/10.1007/s00508-017-1223-x.

4. Petzke F, Kohlmann T. Just one number alone will not suffice: for a differentiating epidemiological collation of chronic pain. Schmerz. 2014;28(5):457-8.

5. Driscoll MA, Kerns RD. Integrated, team-based chronic pain management: bridges from theory and research to high quality patient care. AdvExp Med Biol. 2016;904:131-47.
6. Köppen PJ, DornerTE, Stein KV,SimonJ, Crevenna R.Health literacy, pain intensity and pain perception in patients with chronic pain. Wien Klin Wochenschr. 2017;129. https:// doi.org/10.1007/s00508-017-1301-0.

7. van Hecke O, Torrance N, Smith BH. Chronic pain epidemiology and its clinical relevance. $\mathrm{Br} \mathrm{J}$ Anaesth. 2013;111(1):13-8.

8. Keilani M, Crevenna R, Dorner TE. Sleep quality in subjects suffering from chronic pain. Wien Klin Wochenschr. 2017;129. https://doi.org/10.1007/s00508-017-1256-1.

9. Mittendorfer-Rutz E, Dorner TE. Socio-economic factors associated with the lyear prevalence of severe pain and pain-related sickness absence in the Austrian population. Wien Klin Wochenschr. 2017;129. https://doi.org/10.1007/ s00508-017-1222-y.

10. Stewart WF, Ricci JA, Chee E, Morganstein D, Lipton R. Lost productive time and cost due to common pain conditions in the US workforce. JAMA. 2003;290(18):2443-54. 